\title{
Evaluación de la calidad del huevo marrón comercial del cantón La Troncal, Ecuador
}

\author{
Evaluation of commercial brown egg quality of the county La Troncal, \\ Ecuador
}

Vera-Rodríguez JH, Cepeda Landin WE, Torres-Ajila KM, Bueno-Guallpa EK, Mendoza-López CA, Merchan-Pucha BT et al. Evaluación de la calidad del huevo marrón comercial del cantón La Troncal, Ecuador. Rev Colombiana Cienc Anim. Recia. 2020; 12(2):e771. https://doi.org/10.24188/recia. v12.n2.2020.771

Universidad de Sucre, Colombia

Los autores permiten a RECIA reimprimir el material publicado en él. En caso de que un autor quiera traducir o usar una publicación parcial o completa de nuestro Diario, el autor debe obtener un permiso por escrito del editor de la revista.

Copyright (C) 2020. El (los) autor (es), Revista Colombiana de Ciencia Animal - RECIA. 2020. Este es un artículo de acceso abierto distribuido bajo los términos de Creative Commons Attribution 4.0 (https://creativecommons.org/licenses/by-nc-sa/4.0/), El uso, distribución o reproducción está permitido, siempre que se acrediten al autor original y al propietario del copyright y que se cite la publicación original en esta revista, de acuerdo con la práctica académica aceptada. No se permite el uso, distribución o reproducción que no cumpla con estos términos. 


\section{Evaluación delacalidaddelhuevo marróncomercial del cantón La Troncal, Ecuador}

\section{Evaluation of commercial brown egg quality of the county La Troncal, Ecuador}

José Humberto Vera Rodríguez. M.Sc.

Instituto Superior Tecnológico Enrique Noboa Arízaga. Carrera

DOI: https://doi.org/10.24188/recia.v12.n2.2020.771

Tecnología en Producción Agropecuaria. Calle 4 de noviembre y Loja. La

Troncal, Cañar. Ecuador.

*humbertorichi@hotmail.com

(D) https://orcid.org/0000-0003-3027-059X

Wilson Eder Cepeda Landin. Ing.

Instituto Superior Tecnológico Enrique Noboa Arízaga. Carrera Tecnología en Producción Agropecuaria. Calle 4 de noviembre y Loja. La

Troncal, Cañar. Ecuador.

ecepeda90@hotmail.com

(10) https://orcid.org/0000-0002-3743-8378

\section{Katherine Maribel Torres Ajila. Tnlgo.}

Instituto Superior Tecnológico Enrique Noboa Arízaga. Carrera

Tecnología en Producción Agropecuaria. Calle 4 de noviembre y Loja. La

Troncal, Cañar. Ecuador.

maribeltajila04@gmail.com

(1) https://orcid.org/0000-0002-0391-3038

\section{Evelyn Katiusca Bueno Guallpa. Tnlgo.}

Instituto Superior Tecnológico Enrique Noboa Arízaga. Carrera Tecnología en Producción Agropecuaria. Calle 4 de noviembre y Loja. La

Troncal, Cañar. Ecuador.

evelynbueno97@hotmail.com

(10) https://orcid.org/0000-0002-7673-3564

\section{Carlos Alexander Mendoza López. Tnlgo.}

Instituto Superior Tecnológico Enrique Noboa Arízaga. Carrera

Tecnología en Producción Agropecuaria. Calle 4 de noviembre y Loja. La

Troncal, Cañar. Ecuador.

carlos2019mendoza1@gmail.com

(10) https://orcid.org/0000-0001-5738-9406

\section{Bolivar Taurino Merchan Pucha. Tnlgo.}

Instituto Superior Tecnológico Enrique Noboa Arízaga. Carrera

Tecnología en Producción Agropecuaria. Calle 4 de noviembre y Loja. La

Troncal, Cañar. Ecuador.

bolivarmerchan2018@gmail.com

(10) https://orcid.org/0000-0002-6883-4330

\section{Jennifer Jessenia Carpio Gómez. Tnlgo.}

Instituto Superior Tecnológico Enrique Noboa Arízaga. Carrera Tecnología Agropecuaria. Calle 4 de noviembre y Loja. La Troncal, Cañar. Ecuador.

victor2carpio@gmail.com

(iD) https://orcid.org/0000-0002-4281-0023

\section{Rivera Rivera Doris Mirella. Tnlgo.}

Instituto Superior Tecnológico Enrique Noboa Arízaga. Carrera

Tecnología Agropecuaria. Calle 4 de noviembre y Loja. La Troncal, Cañar.

Ecuador.

mirellarivera27@outlook.com

(C) https://orcid.org/0000-0003-1217-3403

Recepción: 1 junio 2020 Aprobación: 4 Agosto 2020 Publicación: 14 agosto 2020

\section{RESUMEN}

Se evaluó la calidad del huevo marrón comercial del cantón La Troncal, Ecuador. Se estudiaron un total de 1200 huevos (parroquia La Troncal, 400 huevos; parroquia Manuel J. Calle, 400 huevos y parroquia Pancho Negro, 400 huevos). Se realizó el análisis físico externo que incluye peso de huevo, espesor y peso de cáscara, y el análisis físico interno, que incluye diámetro, y altura de clara y de yema. Se clasificaron los huevos según color, peso y forma. Cada parroquia se consideró un tratamiento y las medias de las variables del análisis físico externo y del interno, fueron separadas utilizando la prueba Como citar (Vancouver).

Vera-Rodríguez JH, Cepeda Landin WE, Torres-Ajila KM, Bueno-Guallpa EK, Mendoza-López CA, Merchan-Pucha BT et al. Evaluación de la calidad del huevo marrón comercial del cantón La Troncal, Ecuador. Rev Colombiana Cienc Anim. Recia. 2020; 12(2):e771. https://doi.org/10.24188/recia.v12.n2.2020.771 
de Tukey ( $\mathrm{p} \leq 0.05)$. Los colores 90, 100 y 110, fueron los más frecuentes. Según el peso del huevo, las categorías más frecuentes fueron extragrande (38\%), grande (30\%) y gigante (19\%). Según la forma, la mayor frecuencia fue de huevos ovoides y redondos (92\%), siendo más frecuentes, entre estas dos categorías, los huevos ovoides. Las aves de la parroquia Manuel J. Calle produjeron ( $\mathrm{p} \leq 0.05)$ huevos más grandes, con mayor diámetro y altura de clara, con mayor altura de yema, con mayor peso y espesor de cáscara, color marrón más obscuro y principalmente huevos ovoides, mientras que las demás parroquias produjeron valores levemente inferiores. En conclusión, las parroquias de La Troncal, Ecuador, producen huevos grandes, con buen diámetro y altura de clara, y color francamente marrón, es decir producen huevos de muy buena calidad.

Palabras clave: Análisis externo; análisis interno; calidad; huevo comercial.

\begin{abstract}
The quality of commercial brown egg from the county La Troncal, Ecuador, was evaluated. A total of 1,200 eggs were studied (parish La Troncal, 400 eggs; parish Manuel J. Calle, 400 eggs and parish Pancho Negro, 400 eggs). The external physical analysis was performed, which includes egg weight, thickness and shell weight, and the internal physical analysis, which includes diameter, and height of white and yolk. Eggs were classified according to color, weight and shape. Each parish was considered a treatment and means of variables of the external and internal physical analyses were separated using the Tukey test ( $\mathrm{p} \leq 0.05)$. Colors 90,100 and 110 were the most frequent. According to egg weight, the most frequent categories were extralarge (38\%), large (30\%) and giant (19\%). According to shape, the highest frequency was of ovoid and round eggs (92\%), being more frequent, between these two categories, the ovoid eggs. Birds from Manuel J. Calle parish produced $(\mathrm{p} \leq 0.05)$ larger eggs, with greater diameter and white height, higher yolk height, greater weight and shell thickness, a darker brown color and mostly ovoid eggs, while the other parishes produced slightly lower values. In conclusion, the parishes of La Troncal, Ecuador, produce large eggs, with good diameter and white height, and a really brown color, that is, they produce very good quality eggs.
\end{abstract}

Keywords: External analysis; internal analysis; quality; commercial egg.

\title{
INTRODUCCIÓN
}

El huevo de gallina es un alimento altamente nutritivo (1). Es muy completo, fácil de digerir, rico en proteína, y contiene aminoácidos esenciales, en las proporciones óptimas (2). Por la gran importancia social de este alimento, la avicultura es uno de los sectores ganaderos más importantes (3). El huevo debe ser más que una fuente de proteína y energía económica, debe ser inocuo, palatable y de alta calidad. Al disminuir la calidad del huevo, se reduce la confianza de los consumidores, aumentan los riesgos a la salud y se registran pérdidas económicas significativas (2). Existe una amplia variación en las características de los huevos que se comercializan (4).

El consumidor es cada día más consciente de la calidad y la seguridad alimentaria de los productos que consume. La calidad del huevo se determina por su calidad interna y por la ausencia de defectos externos sobre la cáscara. Las alteraciones de la cáscara van a depender de varios factores: edad, manejo, estrés, nutrición y patologías de la gallina. Estos factores afectan el aspecto externo del huevo y la seguridad alimentaria del consumidor (3). Las exigencias del mercado están generando en la industria avícola la necesidad de medir, cuantificar y estandarizar la calidad del huevo (2).

El objetivo de este estudio fue evaluar la calidad del huevo marrón comercial proveniente de tres parroquias del cantón La Troncal, Ecuador, y clasificarlo según color, peso y forma.

\section{MATERIALES Y MÉTODOS}

Sitio de estudio. El análisis de la calidad del huevo marrón comercial se realizó en tres parroquias del cantón La Troncal

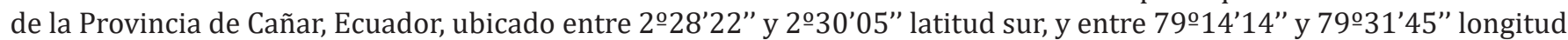
oeste. La jurisdicción cantonal abarca alrededor de 32,780 hectáreas subdivididas en: Parroquia La Troncal 12,483.4 ha, Parroquia Manuel de J. Calle 3,746.8 ha y Parroquia Pancho Negro 16,549.8 ha (Figura 1). La altitud oscila entre 24 y 200 $\mathrm{m}$, con temperaturas promedio $24.6^{\circ} \mathrm{C}$., mínima $20.9^{\circ} \mathrm{C}$ y máxima $29.2^{\circ} \mathrm{C}(5)$. 


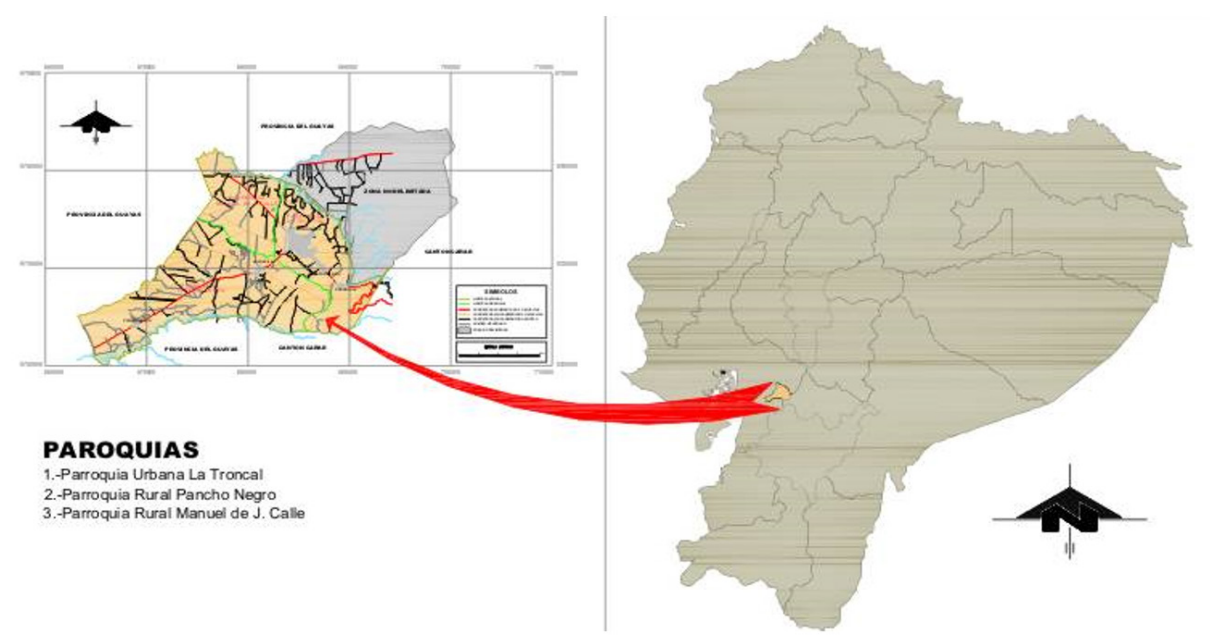

Figura 1. Ubicación geográfica del cantón La Troncal, Ecuador.

Manejo del experimento. Se colectaron un total de 1,200 huevos marrones comerciales procedentes de gallinas ponedoras livianas en el cantón La Troncal: Parroquia La Troncal 400 huevos; Parroquia Manuel de J. Calle 400 huevos y Parroquia Pancho Negro 400 huevos, y se determinaron las siguientes variables:

Análisis físico externo: Peso de huevo (g), diámetro longitudinal (cm), diámetro transversal (cm), índice morfológico, (\%), largo perimetral $(\mathrm{cm})$, ancho perimetral $(\mathrm{cm})$, peso de cáscara $(\mathrm{g})$, porcentaje de cáscara (\%), grosor de cáscara polo ancho $(\mathrm{mm})$, grosor de cáscara polo angosto $(\mathrm{mm})$, grosor de cáscara zona ecuatorial (mm) y espesor de cáscara $(\mathrm{mm})$.

Análisis físico interno: Color de yema, peso de clara (g), peso de yema (g), porcentaje de clara (\%), porcentaje de yema (\%), $\mathrm{pH}$ de clara, $\mathrm{pH}$ de yema, diámetro de clara $(\mathrm{cm})$, diámetro de yema $(\mathrm{cm})$, altura de clara $(\mathrm{cm})$, altura de yema $(\mathrm{cm})$, índice de clara (\%) e índice de yema (\%).

Clasificación por color, peso y forma de huevo: El color se clasificó según la escala Hy-Line International. La escala utilizada tiene valores de 5 a 110, correspondiendo el primer valor al color blanco y el último, al color marrón más oscuro. La clasificación del huevo por peso, se obtuvo según la norma técnica ecuatoriana NTE INEN 1973:2013: supergigante, gigante, extragrande, grande, mediano y pequeño. La clasificación del huevo por forma, se ajustó a los siguientes grupos: alargado, alargado-asimétrico, ovoide, redondo y cónico-asimétrico.

Los pesos (g) se obtuvieron con una balanza digital marca CAMRY EK5055. Las medidas de longitud (mm) se obtuvieron con un micrómetro digital FOWLER N 54-815 calibrado a un rango de hasta 2.5 centímetros. Las medidas de longitud (cm) se obtuvieron con una cinta métrica flexible marca TAYLOR y un calibrador análogo marca SURTEK con una precisión de +-03mm. Obtenidos el diámetro longitudinal y el transversal del huevo, el índice morfológico se obtuvo con la fórmula diámetro transversal×100/diámetro longitudinal. El porcentaje de cáscara se obtuvo con la fórmula peso de cáscara×100/peso de huevo.

El color de yema se determinó con el abanico colorimétrico DSM. El porcentaje de yema se estimó con la fórmula peso de yema $\times 100 /$ peso de huevo, el porcentaje de clara se obtuvo con la fórmula peso de clara×100/peso de huevo, para medir el pH de yema y de clara se usaron tiras reactivas universales de $\mathrm{pH}$, el índice de yema se obtuvo con la fórmula altura de yema×100/diámetro de yema y el índice de clara con la fórmula altura de clara×100/diámetro de clara.

Bajo un diseño completamente al azar, cada parroquia se consideró un tratamiento de 400 repeticiones cada uno, es decir, cada huevo fue considerado una unidad experimental. Las medias de las variables del análisis físico externo y del interno fueron separadas utilizando la prueba de Tukey $(\mathrm{p} \leq 0.05)$, para lo cual se utilizó el programa estadístico InfoStaf versión 2019. 


\section{RESULTADOS}

En la Tabla 1, se muestran los resultados del análisis físico interno y externo. Las aves de Manuel J. Calle produjeron $(\mathrm{p} \leq 0.05)$ valores altos de peso del huevo, y de peso, porcentaje y grosor de la cáscara. Las aves de Pancho Negro produjeron $(\mathrm{p} \leq 0.05)$ los valores más bajos en la mayoría de estas variables. El diámetro de clara, altura de clara y altura de yema fueron altos en los huevos provenientes de Manuel J. Calle y bajos en los de las otras dos parroquias. Los valores obtenidos con los huevos de las otras dos parroquias fueron levemente menores.

Tabla 1. Variables del análisis físico del huevo marrón comercial de gallinas ponedoras livianas, de tres parroquias del cantón La Troncal, Ecuador.

\begin{tabular}{|c|c|c|c|c|c|}
\hline \multirow{2}{*}{ Variables } & \multicolumn{3}{|c|}{ Parroquias del cantón La Troncal } & \multirow{2}{*}{$\mathbf{E E}$} & \multirow{2}{*}{ CV } \\
\hline & La Troncal & Manuel J. Ce & ncho Negro & & \\
\hline \multicolumn{6}{|c|}{ Análisis Físico Externo } \\
\hline Peso del huevo (g) & $65.27^{\mathrm{a}}$ & $66.70^{\mathrm{b}}$ & $65.65^{\mathrm{a}}$ & 0.31 & 9.51 \\
\hline Diámetro longitudinal $(\mathrm{cm})$ & $5.96^{c}$ & $5.83^{\mathrm{b}}$ & $5.74^{\mathrm{a}}$ & 0.02 & 6.30 \\
\hline Diámetro transversal $(\mathrm{cm})$ & 4.50 & 4.51 & 4.48 & 0.01 & 5.81 \\
\hline Índice morfológico (\%) & $75.74^{\mathrm{a}}$ & $77.49^{\mathrm{b}}$ & $89.85^{c}$ & 0.25 & 6.16 \\
\hline Largo perimetral $(\mathrm{cm})$ & $17.15^{\mathrm{c}}$ & $16.50^{\mathrm{a}}$ & $16.90^{\mathrm{b}}$ & 0.04 & 4.25 \\
\hline Ancho perimetral $(\mathrm{cm})$ & $15.10^{\mathrm{b}}$ & $14.41^{\mathrm{a}}$ & $15.15^{\mathrm{b}}$ & 0.03 & 3.19 \\
\hline Peso de cáscara (g) & $8.10^{\mathrm{b}}$ & $8.61^{\mathrm{c}}$ & $7.46^{\mathrm{a}}$ & 0.04 & 10.44 \\
\hline Porcentaje de cáscara (\%) & $12.48^{\mathrm{b}}$ & $13.00^{c}$ & $11.47^{\mathrm{a}}$ & 0.07 & 11.96 \\
\hline Grosor cáscara polo ancho (mm) & $0.41^{\mathrm{b}}$ & $0.51^{c}$ & $0.39^{\mathrm{a}}$ & 0.01 & 23.71 \\
\hline Grosor cáscara polo angosto (mm) & $0.39^{\mathrm{a}}$ & $0.48^{\mathrm{b}}$ & $0.39^{\mathrm{a}}$ & 0.01 & 23.77 \\
\hline Grosor cáscara zona ecuatorial (mm) & $0.40^{\mathrm{a}}$ & $0.49^{\mathrm{b}}$ & $0.40^{\mathrm{a}}$ & 0.01 & 22.72 \\
\hline Espesor de cáscara (mm) & $0.40^{\mathrm{a}}$ & $0.49^{\mathrm{b}}$ & $0.39^{\mathrm{a}}$ & 0.01 & 17.93 \\
\hline \multicolumn{6}{|c|}{ Análisis Físico Interno } \\
\hline Color de yema & $14^{\mathrm{b}}$ & $12^{\mathrm{a}}$ & $12^{\mathrm{a}}$ & 0.06 & 9.46 \\
\hline Peso de clara (g) & $36.10^{\mathrm{a}}$ & $38.70^{\mathrm{b}}$ & $38.22^{\mathrm{b}}$ & 0.24 & 12.73 \\
\hline Peso de yema (g) & $21.08^{c}$ & $19.39^{\mathrm{a}}$ & $19.97^{\mathrm{b}}$ & 0.12 & 11.61 \\
\hline Porcentaje de clara (\%) & $55.26^{\mathrm{a}}$ & $57.98^{\mathrm{b}}$ & $57.98^{\mathrm{b}}$ & 0.16 & 5.65 \\
\hline Porcentaje de yema (\%) & $32.28^{c}$ & $29.10^{\mathrm{a}}$ & $30.58^{\mathrm{b}}$ & 0.14 & 9.04 \\
\hline pH de clara & $10^{\mathrm{b}}$ & $9^{a}$ & $10^{\mathrm{b}}$ & 0.03 & 5.45 \\
\hline pH de yema & $9^{b}$ & $9^{\mathrm{a}}$ & $10^{\mathrm{b}}$ & 0.03 & 6.50 \\
\hline Diámetro de clara $(\mathrm{cm})$ & $10.09^{b}$ & $10.49^{c}$ & $9.72^{\mathrm{a}}$ & 0.06 & 11.19 \\
\hline Diámetro de la yema $(\mathrm{cm})$ & $4.35^{\mathrm{b}}$ & $4.12^{\mathrm{a}}$ & $4.45^{c}$ & 0.02 & 7.12 \\
\hline Altura de clara $(\mathrm{cm})$ & $0.67^{\mathrm{a}}$ & $0.74^{\mathrm{b}}$ & $0.69^{\mathrm{a}}$ & 0.01 & 21.16 \\
\hline Altura de yema $(\mathrm{cm})$ & $1.54^{\mathrm{b}}$ & $1.77^{\mathrm{c}}$ & $1.34^{\mathrm{a}}$ & 0.01 & 11.48 \\
\hline Índice de clara (\%) & $6.71^{\mathrm{a}}$ & $7.09^{b}$ & $7.27^{\mathrm{b}}$ & 0.09 & 25.80 \\
\hline Índice de yema (\%) & $35.57^{b}$ & $42.90^{c}$ & $30.35^{\mathrm{a}}$ & 0.23 & 12.74 \\
\hline
\end{tabular}

a, by cletras distintas en una misma hilera indican diferencias estadísticas según Tukey (0.05); EE error estándar de la media; CV coeficiente de variación. $\mathbf{a}, \mathbf{b}$ and $\mathbf{c}$ different letters in the same row indicate statistical differences according to Tukey ( $\mathrm{p} \leq 0.05$ ); EE standard error of mean; CV coefficient of variation.

La Figura 2 muestra el color de la cáscara de los huevos, por parroquia del cantón La Troncal, según Hy-Line International. Respecto al total de huevos (1,200), el color 100 que se considera el mejor, fue el más frecuente (39\%), seguido del color 90 (31\%) y del color 110 (24\%). La frecuencia de los demás colores fue minoritaria (6\%). Es decir, la mayoría de los huevos estudiados fueron francamente de color marrón.

Los huevos color 90, 100 y 110, se colectaron en La Troncal (96\%), seguida de Manuel J. Calle (97\%) y Pancho Negro (89\%). Los huevos provenientes de la parroquia Manuel J. Calle fueron color marrón más oscuro (100 y 110): 78\% y los de La Troncal y Pancho Negro fueron color marrón menos oscuro (90 y 100): 89\% y 64\%, respectivamente. 


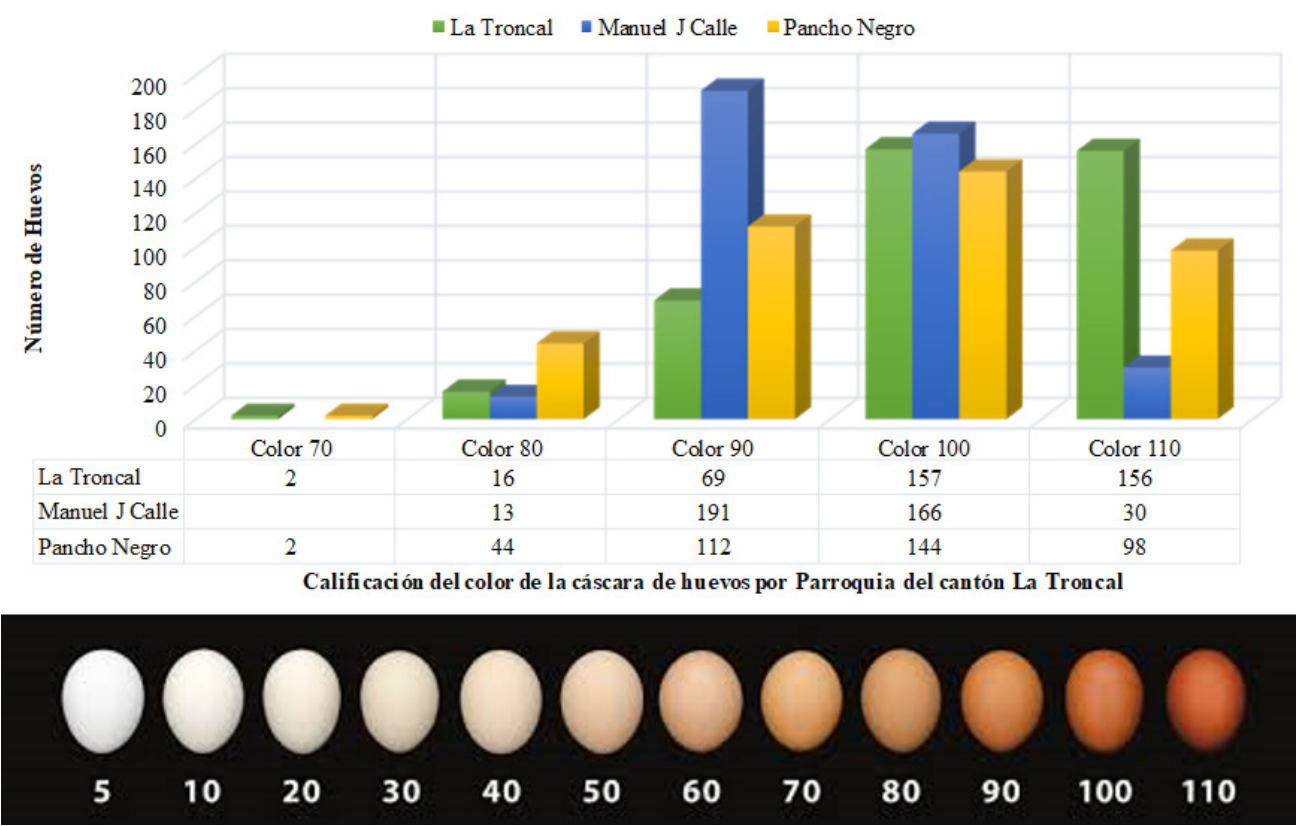

Figura 2. Calificación del color de la cáscara de huevos, por parroquia, cantón La Troncal, Ecuador, según Hy-Line International.

En la Figura 3 se observa la clasificación de los huevos por peso, según la Norma Técnica Ecuatoriana NTE INEN 1973:2013. Se encontraron huevos de cinco o seis categorías de peso en las tres parroquias. Respecto a los 1,200 huevos, la categoría más frecuente fue extragrande (38\%), seguida de grande (30\%), gigante (19\%), supergigante (6\%) y mediano (6\%), la categoría de huevo pequeño fue muy baja (1\%). Es decir $87 \%$ de los huevos fueron de categorías extragrande, gigante y grande.

Por parroquia, La Troncal produjo 79\%, Manuel J. Calle 94\% y Pancho Negro 90\%, de huevos categoría extragrande, gigante y grande.

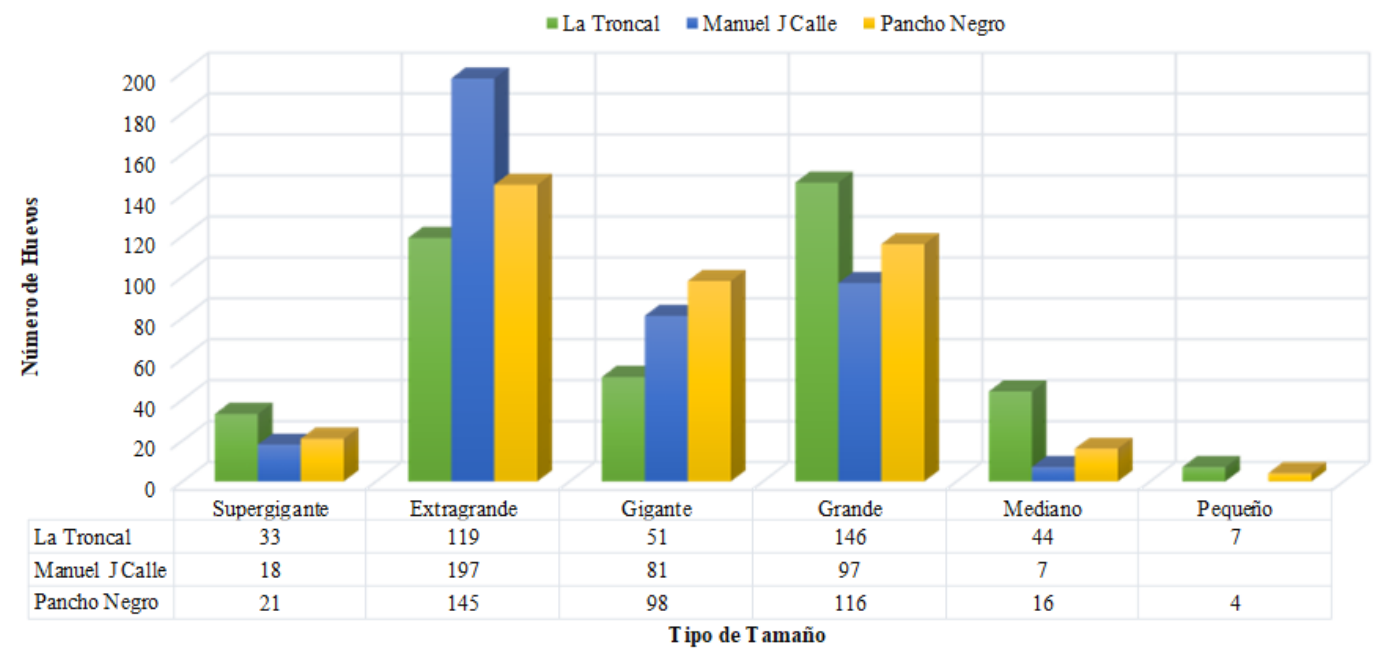

Figura 3. Clasificación de los huevos por peso, por parroquia, cantón La Troncal, Ecuador, según la Norma Técnica Ecuatoriana NTE INEN 1973:2013.

La Figura 4 muestra que, de los 1,200 huevos, 81\% fueron ovoides y 11\% redondos (92\% de estas dos formas). Respecto a los 400 huevos de cada parroquia, de La Troncal, Manuel J. Calle y Pancho Negro se colectaron 72\%, 89\% y 82\% de huevos ovoides, respectivamente. Es decir, las tres parroquias producen principalmente huevos ovoides. 


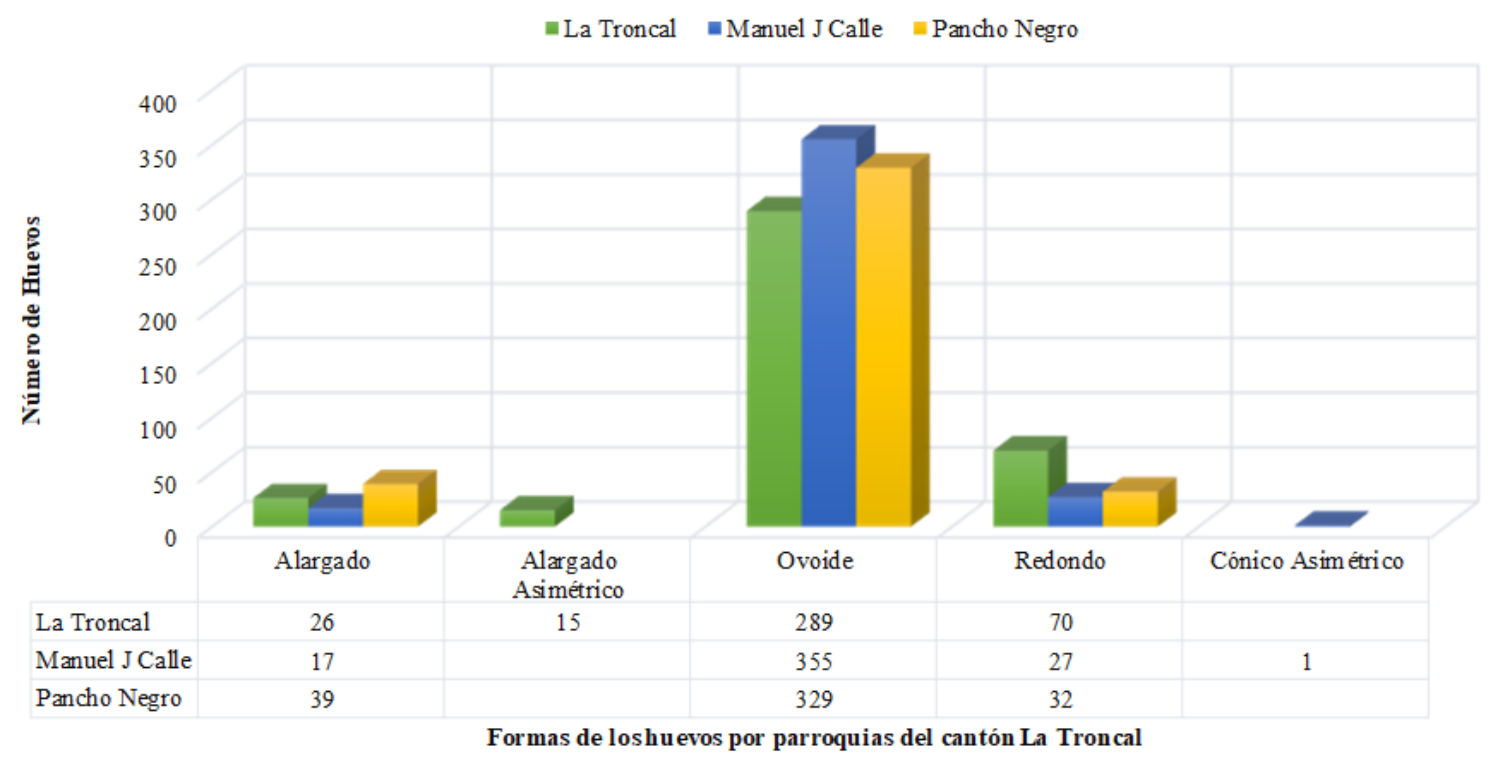

Figura 4. Clasificación de los huevos por forma, por parroquia, cantón La Troncal, Ecuador.

\section{DISCUSIÓN}

Los huevos de gallinas comerciales tienen mayor peso en comparación con los orgánicos y de campo, además los huevos comerciales tienen mayor humedad (6). La edad de las ponedoras tiene un efecto significativo en el peso de huevo, yema, y en el color (7). Rodríguez (8) estudió el peso del huevo en gallinas ponedoras de la línea genética Hy-Line Brown en primera fase de postura y encontró pesos promedios de $56.64 \mathrm{~g}$ al suplementar 1.50 gramos de carbonato de calcio en las últimas horas de la tarde.

Un huevo promedio de gallina pesa entre 60 y 70 gramos, dependiendo de la raza y de la alimentación. El $10.5 \%$ del peso del huevo es cáscara, 31.5\% yema y 58.5\% clara. Las cáscaras de los huevos de gallina pueden tener distintos colores, que dependen de la especie de gallina ponedora y no afectan su calidad, sabor o valor nutricional (9).

El color de la yema es muy importante y depende del contenido de pigmento del alimento, y el tipo de cría y genética de las gallinas ponedoras. Un color muy intenso es bastante demandado en el mercado (10). Comercialmente, el mejor color de yema se encuentra entre 7 y 12 unidades de color (11). El color típico amarillento de la yema de huevo se debe a las xantofilas, un pigmento que no tiene valor nutricional (9). Los huevos con yemas de alta coloración son reconocidos generalmente como procedentes de gallinas sanas y, en consecuencia, los consumidores demandan colores de yema más intensos, la intensidad del color puede considerarse como un indicador de un buen estado de salud, rendimiento y bienestar, y para determinar el color de la yema de forma sencilla y efectiva se debe de contar con el abanico DSM YolkFan ${ }^{\mathrm{TM}}$ como su estándar de calibración (12).

El pH es indicativo del grado de frescura de los huevos, Carranco (13) evaluó el efecto de la temperatura y tiempo de almacenamiento de los huevos, encontrando los siguientes resultados: conservación a $20^{\circ} \mathrm{C}$ : día 0 pH 7.42; día 15 pH 8.70 ; día 30 pH 9.25. Conservación a $4^{\circ} \mathrm{C}$ : día 0 pH 7.42; día 15 pH 8.85; día 30 pH 9.40.

Shiroma (14) evaluó la calidad de los huevos comerciales de expendios tradicionales del distrito de Miraflores, y observó que se vendían huevos con peso promedio de $62.07 \mathrm{~g}$, grosor de cáscara de $0.43 \mathrm{~mm}$, tamaño extra grande y forma globosa o redondeada; el diámetro longitudinal del huevo fue de $5.65 \mathrm{~cm}$, el diámetro transversal $4.38 \mathrm{~cm}$, el índice morfológico $77.84 \%$, el diámetro de yema $4.31 \mathrm{~cm}$ y la pigmentación de yema 5.74 . El mismo autor concluyó que los comercios tradicionales no realizan control de calidad de los huevos comercializados, antes de llegar al consumidor. Por ello, es importante tomar conciencia de que la salud puede verse afectada al consumir huevos de baja calidad.

García (15) reportó para huevos de gallinas White Leghorn L33 de 39 semanas de edad y peso vivo entre 1.5 y 1.6 kg, los siguientes resultados: largo $5.585 \mathrm{~cm}$; ancho $4.265 \mathrm{~cm}$; altura de clara $0.691 \mathrm{~cm}$; ancho de clara $7.183 \mathrm{~cm}$; largo de clara $9.625 \mathrm{~cm}$; altura de yema $1.690 \mathrm{~cm}$; diámetro de yema $4.125 \mathrm{~cm}$; grosor de cáscara $0.41 \mathrm{~mm}$; índice de clara $5 \%$; índice de la yema 4.1\%; índice morfológico $75.55 \%$. 
El espesor de la cáscara del huevo comercial debería estar entre 0.28 y 0.37 mm (11). Rodríguez (8) encontró un grosor de cáscara de $0.40 \mathrm{~mm}$ en huevo de ponedoras Hy-Line Brown que consumieron una dieta con 4.20\% de calcio. Este dato coincide con el estudio de Rodríguez et al (16), quienes encontraron $0.40 \mathrm{~mm}$ de espesor de cáscara en gallinas ponedoras Isa Brown. Las alteraciones de la cáscara afectan la calidad de los huevos destinados al consumo humano (17).

El espesor de la cáscara del huevo está relacionado con la dureza de la misma. El peso de la cáscara se puede medir rompiendo el huevo, separando la cáscara, secándola y expresándolo como porcentaje respecto al peso del huevo. La resistencia de la cáscara no solo depende de su peso y espesor, sino también de la calidad de su estructura. Los principales factores que afectan la calidad de la cáscara son genéticos, nutricionales, productivos y relacionados con el estrés (18).

Al inicio de la producción, los huevos tienen una forma redondeada, que tiende a alargarse conforme avanza el año de puesta, debido a una disminución de la tonicidad muscular de la glándula calcárea en las gallinas de mayor edad (19).

De acuerdo a los resultados obtenidos en la presente investigación, se concluye, que con base en las variables del análisis físico externo y del análisis físico interno, las parroquias estudiadas producen huevos estadísticamente diferentes. Sin embargo, del análisis físico externo, solo tres variables mostraron coeficientes de variación mayores al 20\% (grosor cáscara polo ancho, grosor cáscara polo angosto y grosor cáscara zona ecuatorial) y del análisis físico interno solo dos variables (altura de clara e índice de clara). Es decir, la mayoría de variables mostraron menos del $20 \%$ de coeficiente de variación. Por ejemplo, los rangos de las medias fueron: peso de huevo, $1.43 \mathrm{~g}$; diámetro de clara, $0.77 \mathrm{~cm}$; altura de clara, $0.07 \mathrm{~cm}$; altura de yema, $0.43 \mathrm{~cm}$; peso de cáscara, $1.15 \mathrm{~g}$ y espesor de cáscara, $0.1 \mathrm{~mm}$. Estos rangos o máximas diferencias entre las medias de parroquias, fueron inferiores al 20\%. Es decir, aunque las medias de estas variables entre parroquias, fueron estadísticamente diferentes, sus valores fueron cercanos entre sí.

Además, en el presente trabajo, el valor de espesor de la cáscara, entre otras variables, fue similar a los encontrados por Shiroma (14), García (15), Rodríguez (8) y Rayan (20). Por tanto, el huevo marrón proveniente de las tres parroquias del cantón La Troncal del Ecuador, cumple con la mayoría de las características reportadas en la literatura nacional e internacional, por lo que puede considerarse de buena calidad.

Solamente se sugiere que el huevo se clasifique minuciosamente, para dar cumplimiento a la Norma Técnica Ecuatoriana (NTE INEN 1973:2013), al momento de la compra por el consumidor final.

\section{Conflicto de intereses}

Declaramos no tener conflictos de interés con respecto al trabajo presentado en este informe.

\section{REFERENCIAS}

1. Ivanković M, Mikuš T, Cvrtila Ž. La calidad de los huevos provenientes de las gallinas ponedoras de la cría libre y en jaula. Meso: prvi hrvatski časopis o mesu. 2018; 20(5):419-425. https://doi.org/10.31727/m.20.5.1

2. Saer-Sigismondi A, Causillas Corbella A, Blanco N. ¿Por qué es importante valorar la calidad del huevo? Actualidad Avipecuaria. 2017; 61:20-23. http://bibliotecavirtual.corpmontana.com//handle/123456789/4822

3. Castillo RS, Ródenas JB. Análisis de las alteraciones de la cáscara del huevo de gallina. Nereis. Interdisciplinary Ibero-American Journal of Methods, Modelling and Simulation. 2018; (10):137-147. http://revistas.ucv.es/index. php/Nereis/article/download/388/327

4. Vergara DM, de la Ossa J, Hernández D. Caracterización morfológica de la gallina criolla de traspatio de la subregión Sabana departamento de Sucre (Colombia). Revista MVZ Córdoba. 2019; 24(2):7218-7224. https://doi. org/10.21897/rmvz.1646

5. GAD Municipal La Troncal. Datos generales ciudad La Troncal. Consultado: (01 mayo de 2020). Disponible en: http://www.latroncal.gob.ec/WEB17/VARIOS/CIUDAD.PHP.

6. Quitral V, Donoso ML, Acevedo N. Comparación físico-química y sensorial de huevos de campo, orgánicos y comerciales. Revista Salud Pública y Nutrición. 2009; 10(2):1-10. http://respyn.uanl.mx/index.php/respyn/article/ view $/ 235$ 
7. Sokołowicz Z, Dykiel M, Krawczyk J, Augustyńska-Prejsnar A. Effect of layer genotype on physical characteristics and nutritive value of organic eggs. CyTA-Journal of Food. 2019; 17(1):11-19. https://doi.org/10.1080/19476337.2018 $\underline{1541480}$

8. Rodríguez JHV, Bravo GAH. El Efecto de diferentes niveles de suministro de carbonato de calcio sobre el peso y grosor de la cáscara del huevo. Revista Colombiana de Ciencia Animal-RECIA. 2019; 11(2):719-719. https://doi. org/10.24188/recia.v11.n2.2019.719

9. de las Moras MCR. Alteraciones de la cáscara, clara y yema de huevo. Ganadería. 2008; 52:56-57. https://www. mapa.gob.es/ministerio/pags/biblioteca/revistas/pdf Ganad/Ganad 20085256 57.pdf

10. Bertoncelj J, Gašperlin A, Korošec M. El color de la yema de los huevos de diferentes tipos de producción. Meso: prvi hrvatski časopis o mesu. 2019; 21(4):378-385. https://doi.org/10.31727/m.21.4.4

11. INEN N. Huevos comerciales y ovoproductos. Requisitos. Quito: Copyright. 2013. Consultado (17 mayo de 2020 ). Disponible en: https://www.normalizacion.gob.ec/buzon/normas/nte inen 1973-2.pdf

12. Selecciones Avícolas. La Guía DSM de pigmentación de yema de huevo 2016 y el nuevo Abanico Colorimétrico DSM YolkFan $^{\mathrm{TM}}$ ya están disponibles. Copyright Real Escuela de Avicultura. 2020. Consultado (4 julio de 2020). Disponible en: $\quad$ https://seleccionesavicolas.com/avicultura/2016/04/la-guia-dsm-de-pigmentacion-de-yema-de-huevo2016-y-el-nuevo-abanico-colorimetrico-dsm-yolkfan-ya-estan-disponibles\#: :text=El\%20abanico\%20DSM\%20YolkFan\%E2\%84\%A2,intenso $\% 2$ C $\% 20$ con $\% 20$ referencia $\% 20$ n\%C3\%BAmero $\% 2016$

13. Carranco-Jáuregui ME, Carrillo-Domínguez S, Ávila-González E, Solano MDL._Cambios de la fracción hidrosoluble de huevo de gallinas alimentadas con harina de camarón almacenado a diferentes tiempos y temperaturas. Revista Mexicana de Ciencias Pecuarias. 2017; 8(4):365-373. http://dx.doi.org/10.22319/rmcp.v8i4.4183

14. Shiroma P. Calidad del huevo expendido en los comercios tradicionales y en régimen de autoservicios. Ciencia y Desarrollo. 2019; 22(4):17-21. http://dx.doi.org/10.21503/cyd.v22i4.1833

15. García DM, Colas MC, López WS, Pérez EO, Sánchez AP, Lamazares MC, Grandía RG. El peso corporal y su efecto sobre indicadores bioproductivos en gallinas White Leghorn L33. Revista de la Facultad de Medicina Veterinaria y de Zootecnia. 2016; 63(3):188-200. http://dx.doi.org/10.15446/rfmvz.v63n3.62714

16. Rodríguez JHV, Pinargote MVV, Cedeño JOM. Adición de carbonato cálcico y su repercusión económica sobre el grosor del cascarón en ponedoras. Revista ESPAMCIENCIA. 2012; 3(1):1-7. http://190.15.136.171/index.php/ Revista ESPAMCIENCIA/article/download/46/26

17. Castillo RS, Ródenas JB. Análisis de las alteraciones de la cáscara del huevo de gallina. Nereis. Interdisciplinary Ibero-American Journal of Methods, Modelling and Simulation. 2018; (10):137-147. http://revistas.ucv.es/index. php/Nereis/article/download/388/327

18. Ortiz Á, Mallo JJ. Factores que afectan a la calidad externa del huevo. Albéitar. 2013; 170:18-19. https://norel.net/ en/system/files/factores que afectan a la calidad del huevo.pdf

19. Travel A, Nys Y, Lopes E. Facteurs physiologiques et environnementaux influençant la production et la qualité de l'œuf. INRAE Productions Animales. 2010; 23(2): 155-166. https://productions-animales.org/article/view/3297/10766

20. Rayan GN, Mahrous MY, Galal A, El-Attar AH. Study of some productive performance and egg quality traits in two commercial layer strains. Egyptian Poultry Science Journal. 2013; (33):357-369. http://research.asu.edu.eg/ handle/123456789/155690 